JC-98-06

cond-mat/9812416

\title{
Critical Exponents near a Random Fractal Boundary
}

\author{
John Cardy \\ Department of Physics \\ Theoretical Physics \\ 1 Keble Road \\ Oxford OX1 3NP, UK \\ \& All Souls College, Oxford
}

\begin{abstract}
The critical behaviour of correlation functions near a boundary is modified from that in the bulk. When the boundary is smooth this is known to be characterised by the surface scaling dimension $\tilde{x}$. We consider the case when the boundary is a random fractal, specifically a self-avoiding walk or the frontier of a Brownian walk, in two dimensions, and show that the boundary scaling behaviour of the correlation function is characterised by a set of multifractal boundary exponents, given exactly by conformal invariance arguments to be $\lambda_{n}=\frac{1}{48}(\sqrt{1+24 n \tilde{x}}+11)(\sqrt{1+24 n \tilde{x}}-1)$. This result may be interpreted in terms of a scale-dependent distribution of opening angles $\alpha$ of the fractal boundary: on short distance scales these are sharply peaked around $\alpha=\pi / 3$. Similar arguments give the multifractal exponents for the case of coupling to a quenched random bulk geometry.
\end{abstract}


The subject of boundary critical behaviour [1] is by now well understood, particularly in two dimensions [2]. The two-point correlation function $\langle\phi(r) \phi(R)\rangle$ of a scaling operator $\phi$, which behaves in the bulk at large distances at the critical point as $|r-R|^{-2 x}$, where $x$ is the bulk scaling dimension of $\phi$, is modified when one of the points ( say $r$ ) is close to the boundary to the form

$$
\langle\phi(r) \phi(R)\rangle \sim|r|^{-x}|R|^{-x}|R / r|^{-\tilde{x}}
$$

where $\tilde{x}$ is the corresponding boundary scaling dimension, and the angular dependence has been suppressed for clarity. In two dimensions, the role played by $\tilde{x}$ is emphasised by making the conformal mapping $z \rightarrow \ln z$ of the upper half plane to a strip of width $\pi$ : in that geometry the correlation function decays exponentially along the strip with an inverse correlation length equal to $\tilde{x}[3]$.

Eq. 1 refers to the case when the boundary is smooth (at least on scales $\ll r)$ and it is an interesting to ask whether these results are modified when the boundary is a fractal on these scales. The example of an edge (or corner in two dimensions) on the boundary was analysed some time ago [4] and it was shown that new edge scaling dimensions arise which depend continuously on the opening angle $\alpha$. In two dimensions [5] this dependence is given by conformal invariance arguments by the simple form $x(\alpha)=\pi \tilde{x} / \alpha$. This suggests that close to a fractal boundary, which may be thought of as presenting a distribution of opening angles (which perhaps also depends on the scale at 
which it is probed), an even more complicated behaviour should obtain.

In the case of a random fractal, one also expects to see behaviour characteristic of correlation functions in a quenched random environment. That is, they may exhibit multiscaling, which means that the average of their $n$th power does not scale in the same way as the $n$th power of their average. In this letter, we consider two cases where this problem is exactly solvable using conformal invariance methods in two dimensions, namely when the fractal boundary is a self-avoiding walk, and when it is the frontier (exterior boundary) of a Brownian (ordinary) random walk. In fact, both cases turn out to give identical results. Our methods are a simple generalisation of arguments due to Lawler and Werner [6], who have derived exact relations between multifractal exponents corresponding to self-intersection properties of Brownian walks in two dimensions. This corresponds to the special case when $\phi$ is a free scalar field satisfying Laplace's equation. This physically interesting example, and its relation to the exponents of star polymers, was in fact discussed some time ago in $4-\epsilon$ dimensions by Cates and Witten [7]. The results of Lawler and Werner have recently been given an elegant interpretation and derivation by Duplantier [8] in the context of coupling the system to a randomly fluctuating metric.

Consider for definiteness self-avoiding walks $\gamma$ which are constrained to pass through the origin $O$. In order to be able to apply conformal invariance arguments, we work in the fixed fugacity ensemble, in which each walk of 
length $L$ is counted with a weight $y^{L}$, at the critical point where $y^{-1}=\mu$, the lattice-dependent connective constant. The properties of the measure on walks on distance scales much larger than the lattice spacing are then supposed to be conformally invariant. Denote the radial coordinate by $\rho$. We want to focus on those walks which have a typical linear size $R$, and for which the origin $O$ is a typical interior point. Without loss of generality for computing scaling dimensions, we may then restrict the walks $\gamma$ to have the form of a pair of mutually avoiding self-avoiding walks, starting from $O$ and ending on the circle $\rho=R$. In the same spirit we may take these points to be the first intersections of the walks with this circle. The region bounded by $\gamma$ and an arc of the circle $\rho=R$ is thus simply connected. In this region we consider a critical system (for example an Ising model) with a suitable conformally invariant boundary condition [2] on $\gamma$ and on $\rho=R$ (for example, that the spins are free). Consider the correlation function $\left\langle\phi(r) \phi\left(R^{\prime}\right)\right\rangle$, where, without loss of generality for computing scaling dimensions [7] we can choose $\left|R^{\prime}\right| \sim R$, and we are interested in the limit where $r \ll R$. The geometry is illustrated in Fig. 11.

Obviously this correlation function depends on $\gamma$, but we may hope to be able to compute suitable averages of this quantity over realisations of $\gamma$. By analogy with the case of a smooth boundary, we expect that

$$
\overline{\left\langle\phi(r) \phi\left(R^{\prime}\right)\right\rangle^{n}} \sim|r|^{-n x}|R|^{-n x}|R / r|^{-\lambda_{n}},
$$

where the overline means an average over all the allowed realisations of $\gamma$. 


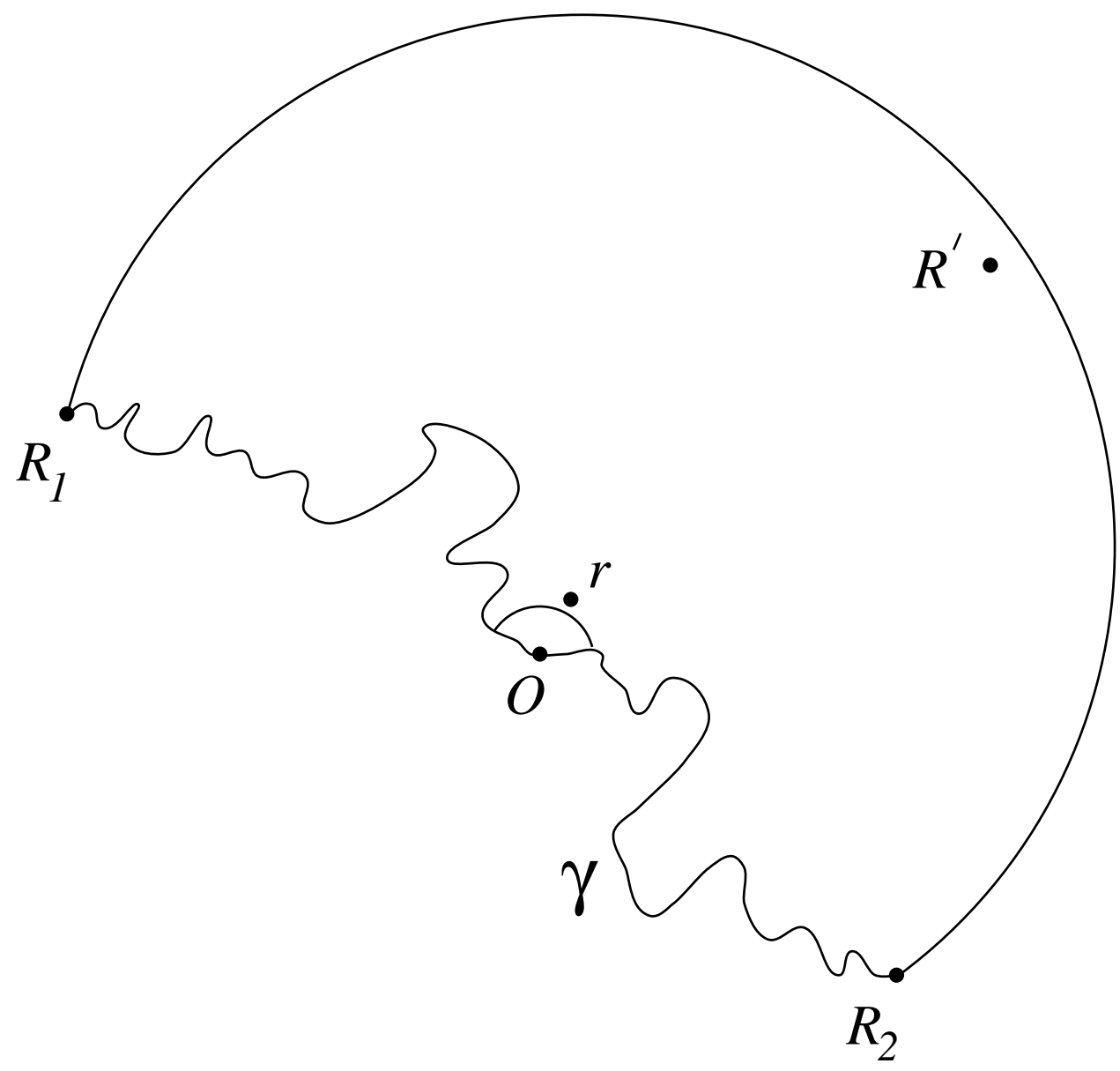

Figure 1: Geometry in which the simply connected region $R_{1} O R_{2}$ is bounded by a self-avoiding walk $\gamma$ and an arc of the circle $\rho=R$. This region contains a critical system of which the correlation function of local operators at points $r$ and $R^{\prime}$ is of interest. This region, excluding the disc $\rho<|r|$, is conformally mapped into a long strip of width $\pi$ and length $\ell_{\text {eff }}(\gamma, r / R)$. 
Note that if the average were over smooth boundaries only, we would expect $\lambda_{n}=n \tilde{x}$, with only the prefactor modified by the averaging.

We now make a conformal transformation which maps the fractal boundary into a smooth one. It is convenient to exclude the disc $\rho<r$. This leaves the simply connected region bounded by two segments of $\gamma$ and arcs of the circles $\rho=r$ and $\rho=R$. By the Riemann theorem, the interior of this region may be mapped conformally by an analytic function $z \rightarrow f(\gamma,|r|, R ; z)$ onto the interior of a strip of width $\pi$, but with a length which is not simply $\ell \sim \ln (R / r)$, but which will also depend on $\gamma$. Let us denote this by $\ell_{\mathrm{eff}}(\gamma, \ell)$. The correlation function $\left\langle\phi(r) \phi\left(R^{\prime}\right)\right\rangle$ will be related by this conformal mapping to one between operators located near the ends of this strip. Taking the $n$th power and averaging we see by comparison with (2) that

$$
e^{-\lambda_{n} \ell} \sim \overline{e^{-n \tilde{x} \ell_{\mathrm{eff}}(\gamma, \ell)}}
$$

At this point, we need further information about averages of quantities of the form $e^{-p \ell_{\mathrm{eff}}}$, for arbitrary $p$. In particular, let us consider the case where $n=1$, and $\phi(r)$ is the $M$-leg operator for $M$ mutually self-avoiding walks. It is convenient to generalise slightly and take $\phi\left(R^{\prime}\right)$ to be a product of distinct single leg operators corresponding to the walks all ending on the arc of the circle $\rho=R$. The correlation function then gives the number of such walks which all begin at $r$ and end at a distance $\sim R$, and, in the critical fugacity ensemble, scales in the bulk like $|r-R|^{-x_{M}-M x_{1}}$, and near a smooth boundary according to (1) with a boundary scaling dimension $\tilde{x}_{M}$. 
Coulomb gas arguments [9] lead to the conjectures $x_{M}=\frac{3}{16} M^{2}-\frac{1}{12}$ and $\tilde{x}_{M}=\frac{1}{8} M(3 M+2)$, which have been confirmed by numerical work and various other known exact information. If we now imagine taking an $M$-leg star polymer near the fractal boundary $\gamma$, which itself is a 2-leg star polymer, and performing the same average over the realisations of $\gamma$, the result will be an $(M+2)$-leg star polymer in the bulk. We conclude that

$$
e^{-\left(x_{M+2}-x_{2}\right) \ell} \sim \overline{e^{-\tilde{x}_{M} \ell_{\mathrm{eff}}(\gamma, \ell)}}
$$

where the factor $e^{x_{2} \ell}$ arises from the normalisation of the probability distribution of $\gamma$. Note that although this is initially defined only for $M$ a non-negative integer, it may be continued to other real values for which the average exists. Comparing with (3) we may therefore choose $M$ such that $\tilde{x}_{M}=n \tilde{x}$, solve for $M$ using the exact conjecture for $\tilde{x}_{M}$ given above, and substitute this into the exact form for $x_{M+2}$. After some simple algebra, this gives the result for $\lambda_{n}$ quoted in the abstract.

A similar argument may be made when $\gamma$ is the exterior boundary of a Brownian walk. In this case the measure is rigorously known to be conformally invariant. As in the example considered by Lawler and Werner [6], one may now invoke the exact conjectures made by Duplantier and Kwon [10] for the relevant dimensions of $M$ mutually avoiding ordinary random walks. However, the final result is identical, indicating that there is strong element of universality between these two fractals, not only with respect to their fractal dimensions 11]. 
The nontrivial dependence of $\lambda_{n}$ shows that correlation functions near the boundary have a broad distribution of values. In particular, their average value, which scales as $(r / R)^{\lambda_{1}}$, may be quite different from a typical value. As argued, for example, in Ref. [12], the typical dependence should be of the form $(r / R)^{\lambda^{\prime}}$, where $\lambda^{\prime}=d \lambda_{n} /\left.d n\right|_{n=0}=3 \tilde{x}$. Interesting enough this is the behaviour which would obtain in wedge of interior angle $\alpha=\pi / 3$. This idea may be made more explicit by interpreting (3) in terms of an average over a scale-dependent distribution $P(\alpha, \ell)$ of interior opening angles:

$$
\int_{0}^{2 \pi} d \alpha P(\alpha, \ell) e^{-(\pi / \alpha) n \tilde{x} \ell} \sim e^{-\lambda_{n} \ell}
$$

Requiring that this be valid for all positive real $n$ determines the form of $P$. First we see that the behaviour of $\lambda_{n} \sim n \tilde{x} / 2$ as $n \rightarrow \infty$ at fixed $\ell$ implies that the effective angle in this regime is $\alpha \sim 2 \pi$. This is in agreement with a general argument of Cates and Witten [7]. If we set $\omega \equiv \frac{\pi}{\alpha}-\frac{1}{2}$ and $u \equiv n \tilde{x}$, and define $\widetilde{P}(\omega, \ell) d \omega=P(\alpha, \ell) d \alpha$ the above equation simplifies to

$$
\int_{0}^{\infty} d \omega \widetilde{P}(\omega, \ell) e^{-\omega u \ell} \sim \exp (-(5 \ell / 24)(\sqrt{1+24 u}-1)) \quad .
$$

Making the ansatz $P(\omega, \ell) \sim e^{5 \ell / 24} e^{-\ell(a \omega+b / \omega)}$ and using steepest descent then leads to the solution

$$
\widetilde{P}(\omega, \ell) \sim \exp \left(-\frac{\ell}{24}\left(\sqrt{\omega}-\frac{5}{2 \sqrt{\omega}}\right)^{2}\right),
$$

valid for large $\ell$ (i.e. $r / R \ll 1$ ) and where we have suppressed more slowly varying prefactors. Note that this result is independent of $\tilde{x}$, consistent with 
it being an intrinsic property of the fractal' It shows that the effective opening angle has a broad distribution which, however, becomes more and more strongly peaked around the typical value $\omega=\frac{5}{2}\left(\alpha=\frac{\pi}{3}\right)$ as $r / R \rightarrow 0$, with a width of order $(\ln (R / r))^{-1 / 2}$.

A similar calculation may be carried out when the point $O$ is the root of an $N$-leg star polymer, by replacing $x_{M+2}-x_{2}$ in (4) by $x_{M+N}-x_{N}$. The case $N=1$ gives the end multifractal exponents, which, as first pointed out by Cates and Witten, are different form those which arise when $O$ is a typical interior point.

In the case considered by Cates and Witten [7], where $\phi$ is a massless scalar field (with bulk scaling dimension $x=0$ ) satisfying Dirichlet conditions $\phi=0$ on the boundary, the appropriate boundary scaling operator is $\partial_{\perp} \phi$ with dimension $\tilde{x}=1$. In that case we find that $\lambda(1)=\frac{2}{3}=2-D$, where $D=\frac{4}{3}$ is the fractal dimension of the boundary. This is a consequence of the fact that $\phi$ satisfies Laplace's equation, equivalent to the conservation of particle flux in the Brownian interpretation [7]. Note that we were lead to this unique result from making simple assumptions (rigorously grounded in the Brownian case) about the conformal invariance of the measure on $\gamma$. This suggests that all such curves which, with probability one, bound a simply connected region when viewed on macroscopic distance scales will fall

\footnotetext{
${ }^{1}$ However, it should be noted that the method of averaging used here, which sums over all realisations of $\gamma$ passing through a given point $O$ at given distance $r$ from a fixed point, tends to emphasise those parts of the fractal for which the opening angle is small.
} 
into this universality class and, in particular, will have $D=\frac{4}{3}$.

One of the most interesting features of our main result is that the $\lambda_{n}$ are generally, even for integer $n$, irrational (but algebraic) numbers. This is not in disagreement with any established results, since even if the bulk critical theory is unitary, there is no reflection positivity in the presence of a fractal boundary and so the theorem of Friedan, Qiu and Shenker [13] is evaded. However, most examples of exactly calculable critical exponents in two dimensions have, even in nonunitary cases, led to rational values. Recently Duplantier [B] has given an interesting interpretation of these type of results in the case of a general mixture of Brownian and self-avoiding walks, by considering the effects of coupling the system to a fluctuating background metric (quantum gravity). He was able to argue that, just as the scaling dimensions of overlapping objects in flat space should be added to obtain that of the composite, when they are coupled to quantum gravity their dressed scaling dimensions are additive if they avoid each other. In this way, by going back and forth between flat space and quantum gravity, and using the relation between ordinary and dressed scaling dimensions first obtained by Knizhnik, Polyakov and Zamolodchikov (KPZ) [14], specialised to the case $c=0$ appropriate to Brownian walks, he was able not only to recover the results of Lawler and Werner [6], but also to derive the earlier conjecture of Duplantier and Kwon [10]. Thus, for this example, his methods are more powerful than the simple arguments we have used above, since we 
found it necessary to invoke the conjectured values for the $M$-leg scaling dimensions. Since our main result is a simple generalisation to the case when $\tilde{x} \neq 1$, one would expect that similar quantum gravity methods might apply. However, our result is supposed to be valid for a bulk theory with arbitrary central charge $c$, and it is therefore not clear why the KPZ relation with $c=0$ should appear in this more general case.

We have given an exact formula for the multiscaling boundary exponents of an arbitrary conformally invariant two-dimensional critical system close to a random fractal boundary. This is the first example when such a multifractal spectrum with a non-trivial analytic structure has been found exactly. The basic method was to realise that this kind of geometric quenched disorder may be gauged away by making a suitable conformal transformation, at the cost of modifying the moduli (in this case $\ell=\ln (R / r)$.) The effective distribution of $\ell_{\text {eff }}$ is then probed by replacing the critical system by one with $c=0$ (in our case, self-avoiding walks) for which the partition function is unity and therefore the quenched average of a correlation function is the same as its annealed average, which is more simply dealt with.

Similar ideas may be applied to a critical system coupled to bulk quenched disorder in the form of a random metric (which may be realised as the continuum limit of a randomly connected lattice.) In this case we may consider an annulus of inner and outer radii $r$ and $R$ respectively, which in the case of a flat metric may be mapped conformally by the transformation $z \rightarrow \ln z$ 
to a flat metric on a cylinder of perimeter $2 \pi$ and length $\ell=\ln (R / r)$. The inverse correlation length along this cylinder is then equal to the bulk scaling dimension $x$ [3]. An arbitrary metric $g$ on the annulus is also conformally equivalent to the cylinder with a flat metric but with a length $\ell_{\text {eff }}(g, \ell)$. In analogy with (3) the multifractal bulk exponents $\lambda_{n}^{b}$, which govern the decay of the quenched average of the $n$th power of the bulk correlation function, are given by

$$
e^{-\lambda_{n}^{b} \ell} \sim \overline{e^{-n x \ell_{\mathrm{eff}}(g, \ell)}}
$$

We now consider the special case when the critical system case has $c=$ 0 . In that case the quenched and annealed averages are identical, and the respective scaling dimensions $X_{0}$ and $X$ of an operator in flat space and when coupled to a fluctuating metric are related by the $c=0$ version of the KPZ relation [14] $X_{0}=\frac{1}{3} X(1+X)$. Thus if we now set $X_{0}=n x$ and solve for $X$, this will yield $\lambda_{n}^{b}$. The result is

$$
\lambda_{n}^{b}=\frac{1}{2}(\sqrt{1+12 n x}-1)
$$

This result for the scaling dimensions when coupled to a quenched random lattice, was derived for the case $n=1$ by Baillie, Hawick and Johnston [15], but in fact these correlation functions exhibit multiscaling, with a whole spectrum of such exponents. Note that in this case the typical decay of a correlation function is determined by $\left(\lambda^{b}\right)^{\prime}=3 x$.

The author thanks G. Lawler and B. Duplantier for explaining their ideas, and the Fields Institute, Toronto, where this work was started, for its hospi- 
tality. This research was partly supported by the Engineering and Physical Sciences Research Council under Grant GR/J78327.

After this work was completed preprints by Duplantier [16] and Aizenman, Duplantier and Aharony [17] appeared in which, among other things, it is argued that the fractal dimension of the accessible perimeter of a per-

colation cluster has fractal dimension $D=\frac{4}{3}$, consistent with the general arguments advanced above.

\section{References}

[1] See, for example, H. W. Diehl in Phase Transitions and Critical Phenomena, v. 10, C. Domb and J. L. Lebowitz, eds., (Academic, 1986).

[2] J. L. Cardy, Nuclear Physics B240 [FS12], 514-532, 1984; Boundary Conditions in Conformal Field Theory, proceedings of the Taniguchi Symposium on Integrable Models, Kyoto, 1988, Adv. Studies in Pure Math., 19, 127, 1989.

[3] J. L. Cardy, J. Phys. A17, L385, 1984.

[4] J. L. Cardy, J. Phys. A16, 3617, 1983.

[5] J. L. Cardy, Nuclear Physics B270, 186, 1986.

[6] G. F. Lawler and W. Werner, to be published. 
[7] M. E. Cates and T. A. Witten, Phys. Rev. Lett. 56, 2497, 1986; Phys. Rev. B 35, 1809, 1987.

[8] B. Duplantier, Phys. Rev. Lett. 81, 5489, 1998; Phys. Rev. Lett., to be published (cond-mat/9812439).

[9] B. Duplantier and H. Saleur, Nucl. Phys. B 290, 291, 1987.

[10] B. Duplantier and K.-H. Kwon, Phys. Rev. Lett. 61, 2514, 1988.

[11] B. Mandelbrot, The Fractal Geometry of Nature (Freeman, New York, 1982).

[12] A. W. W. Ludwig, Nucl. Phys. B 330, 639, 1990.

[13] D. Friedan, Z. Qiu and S. Shenker, Phys. Rev. Lett. 52, 1575, 1984.

[14] V. G. Knizhnik, A. M. Polyakov and A. B. Zamolodchikov, Mod. Phys. Lett. A 3, 819, 1988.

[15] C. F. Baillie, K. A. Hawick and D. A. Johnston, Phys. Lett. B 328, 284, 1994.

[16] B. Duplantier, cond-mat/9901008.

[17] M. Aizenman, B. Duplantier and A. Aharony, cond-mat/9901018. 